\title{
Compañía, buenos días, de pie. Construcción del cuerpo militar ${ }^{*}$
}

\author{
María Camila Otálora* \\ Recibido: noviembre 26 de 2015 • Evaluado: diciembre 5 de 2015
}

Aceptado: enero 15 de 2016

\section{Resumen}

Este trabajo se desarrolla en la Escuela Militar de Cadetes José María Córdova en Bogotá, donde a partir de un trabajo etnográfico de observación participante, entrevistas y captura de imágenes durante más de un año, se responde a la inquietud sobre cómo preparan los cadetes sus cuerpos en la institución hasta alcanzar el título de subtenientes del Ejército Nacional. La institución se encuentra en un momento coyuntural de cambios relacionados al posconflicto y al respeto de los derechos humanos que han transformado la estructura de enseñanza militar. De acuerdo a las nuevas necesidades de la institución, se busca una formación integral que le enseñe a los cadetes formas básicas como comer, dormir, bañarse, el manejo estratégico de las armas y la formación académica universitaria, todo esto durante cuatro años de educación permanente, donde el cuerpo de los jóvenes se constituye como reflejo del cuerpo del Ejército.

Palabras clave: ejército; cuerpo; educación militar; cadete; etnografía

\footnotetext{
"Investigación etnográfica realizada en enero de 2014 y finalizada en abril de 2015, con la colaboración de la Facultad de Administración Logística de la Escuela Militar de Cadetes José María Córdova, presentada en el II Encuentro Latinoamericano de investigadores/as sobre el cuerpo y corporalidades en las culturas, Bogotá, octubre de 2015. DOI: http://dx.doi. org/10.15332/s2248-4418.2016.0001.01

** Antropóloga con énfasis en salud de la Pontificia Universidad Javeriana Bogotá, correo:kmixox@gmail.com
} 


\section{Company, good morning, standing at attention. Construction of the military body}

\section{Abstract}

This work takes place in Escuela Militar de Cadetes Jose Maria Cordova in Bogota, where from an ethnographic work of participant observation, interviews and image capture for more than a year (2014-2015), responds to concerns on how the cadets prepare their bodies in the institution to achieve the title of second lieutenants of the National Army. The institution is in a cyclical time related to post-conflict and respect for human rights that have transformed the structure of military education according to the new needs of the institution. Comprehensive training is currently looking in order to teach the cadets basic ways such as eating, sleeping, bathing, strategic use of arms and university training, all during four years of ongoing formation where the body of the young men is constructed as a reflection of the Army.

Keywords: army; body; military education; cadet; ethnography 


\section{Introducción}

El oficio de la guerra, también llamado arte de la guerra (Montgomery, 1969) es ejecutado por un grupo poblacional específico que se forma para su ejecución. Este, por lo menos, es el caso de los oficiales militares colombianos que se profesionalizan en el oficio desde el entrenamiento corporal e intelectual sobre las técnicas propias de la guerra. El aprendizaje de este oficio se hace por medio de la intervención corporal, donde el futuro oficial se apropia de los elementos necesarios no solo para desempeñarse en los escenarios bélicos, sino también en los escenarios sociales propios de la comunidad militar que les exigen a sus participantes comportarse, verse y reconocerse como militares.

Para entender la transformación que hace un futuro oficial al entrar a la comunidad castrense, se propone la siguiente pregunta: ¿Cómo prepara un cadete su cuerpo para el oficio militar en la Escuela Militar de Cadetes José María Córdova? Esta pregunta se interesa en la descripción de los inicios de la vida militar, donde se recibe formación explícita sobre la utilización del cuerpo; también tiene como fin comprender las transformaciones a las que son expuestos los y las jóvenes cadetes durante sus cuatro años de formación dentro de la Escuela Militar, pues está planteada de manera que la acción de preparación recae sobre los cadetes, porque aunque la institución diseña y ejecuta la formación, es el cadete quien libremente decide dejarse permear y competir para adaptarse a ella.

La introducción al mundo militar puede verse desde diversos aspectos de la vida en comunidad, puesto que la formación del cadete está pensada para educarlo de manera integral, desde la manera en que come hasta la manera en que aprende a utilizar las armas. Por esta razón, este trabajo pretende entender estos procesos a partir del estudio del cuerpo del cadete, que es el primer foco de atención dentro de la formación como militar.

Una de las principales razones de la elección de este tema como objeto antropológico es el cambio al que se han visto expuestas las Fuerzas Militares en las últimas décadas: debido al ingreso de los derechos humanos como directriz de la pedagogía castrense y a la especialización académica que han buscado las instituciones estatales, antiguos sistemas de formación basados en la vergüenza pública, los ritos de paso dolorosos, las sesiones exageradas de ejercicio propias de las comunidades militares - y que son relatadas por oficiales retirados-, en la actualidad se consideran obsoletas y se han remplazado por medios distintos de formación y cohesión militar. 
Los sistemas militares de formación, utilizados desde tiempos inmemoriales por diferentes culturas para la garantía de la seguridad estatal, han sido empleados en diferentes instituciones de formación como colegios, empresas, centros de reclusión, conventos, entre otros, esto a causa del gran componente físico que tiene la pedagogía militar, pues permite el ordenamiento eficaz de los cuerpos; esto quiere decir que el Ejército desde siempre ha sido un modelo de educación física que refleja la necesidad de moldear los cuerpos en función de objetivos institucionales, en este caso, en función del Estado.

El tema del cuerpo se introdujo de manera privilegiada en la disciplina antropológica en el siglo XIX; desde ese momento, el cuerpo se ha visto desde diferentes lugares dentro de la antropología (Ayús y Eroza, 2008), por lo cual es importante resaltar, antes que consideralo una obviedad, que el lugar de análisis del cuerpo para este trabajo parte de lo que se conoce como la antropología social o cultural, no desde perspectivas fenomenológicas, de darwinismo social o que tengan como centro a la filosofía del cuerpo.

En el siglo XIX, el tema del cuerpo se abordó desde la materialidad visible de este en diferentes disciplinas, pero es en el siglo XX donde toman fuerza las perspectivas que comprenden al cuerpo desde la condición humana como un hecho emocional, afectivo y libidinal, es decir, desde perspectivas que entienden al cuerpo desde su sensibilidad. Producto de estas formas de abordar el cuerpo es el auge de los estudios del cuerpo en las últimas tres décadas, derivados del interés en cuestionar las certezas que se creían tener sobre el cuerpo material (Pedraza, 2007).

El cuerpo será reconocido en este trabajo como un espacio social del individuo con el cual experimenta el mundo, entendiendo así que "las concepciones que se forman acerca del cuerpo humano (como el cuerpo mismo) son meollos receptores, ordenadores y proyectores de las esferas físicas y sociales que las envuelven" (López, 1996, p. 7).

Colombia es un lugar interesante para la realización de investigaciones sobre las Fuerzas Armadas, ya que el país se encuentra en un momento de conflicto permanente entre las organizaciones armadas estatales y no estatales, que ha producido diferentes relaciones entre la milicia y la población civil $^{1}$. Entre los trabajos hechos desde las ciencias sociales para el estudio de

1 Durante el desarrollo del texto se utiliza la categoría de "población civil”, “civiles” o "civil" para identificar a todos quienes no pertenecen a las fuerzas armadas. 
las fuerzas armadas destaco los de Torres (2000) y Castañeda (2006), que se han acercado a las Fuerzas Militares como sujetos relevantes en el estudio histórico y social del país, más allá de las dimensiones estereotipadas que vinculan al Ejército únicamente con temas de conflicto bélico.

La milicia, como ente directamente dependiente del Estado, debe regirse por los cambios que este propone, es así como la modernización nacional trajo consigo propuestas educativas especializadas, la adopción de los derechos humanos y del derecho internacional humanitario, la implementación de nuevas formas de derecho castrense regidas por cánones internacionales y estéticas menos rudas para los oficiales. Es por esto que esta investigación tiene una pertinencia histórica importante, ya que con el posible fin del conflicto interno, las políticas de formación militar serán, a futuro, muy distintas de las que se conocen hoy; es por esto que este trabajo puede constituir algunas de las últimas observaciones de la formación militar antes de la firma de los acuerdos de paz desarrollados en La Habana para la terminación del conflicto interno entre el Estado colombiano y las Fuerzas Armadas Revolucionarias de Colombia.

\section{Metodología}

Para entender los procesos de intervención corporal en los y las cadetes, se realizó un trabajo etnográfico a partir de la participación cotidiana en sesiones de instrucción y entrenamiento militar, procesos de incorporación, fiestas, ceremonias, visitas, ensayos y en otros espacios institucionales como comedores, aulas, auditorios, baños, gimnasios, pistas de entrenamiento y casinos. Este proceso etnográfico estuvo apoyado por la realización de entrevistas a cadetes, oficiales y personas vinculadas con la formación militar, y la toma de videos y fotografías dentro de la institución durante más de un año de asistencia continua.

El cambio de los cadetes se produce paulatinamente durante cuatro años de formación, en los que se encuentran internos en la institución; para observar esta transformación, se realiza una observación simultánea durante catorce meses de cada uno de los niveles, esto a partir de la interacción con cada nivel de formación.

El trabajo se estructura en cuatro fases de investigación: la primera, de acercamiento al campo y la segunda, de observación participante; estas dos 
primeras fases permiten realizar un proceso descriptivo de los inicios de la vida militar. La tercera fase se centra en la realización de entrevistas y la cuarta, en evaluación y análisis de resultados dentro y fuera de la institución. Las cuatro fases del trabajo estuvieron apoyadas por la toma continua de material videográfico y la socialización de resultados con algunos de los participantes.

Se realizaron entrevistas semiestructuradas con diez cadetes por nivel de formación, de diferentes compañías, y el seguimiento semanal por medio de entrevistas abiertas de dos cadetes escogidos aleatoriamente durante la investigación por cada nivel de formación. Se entrevistaron de manera abierta trece oficiales vinculados con la formación institucional. De todos los encuentros se realizaron grabaciones, de las cuales se hicieron transcripciones selectivas de 72 horas y media de grabación, que a su vez se sistematizaron en softwares de análisis cualitativo junto con la clasificación y estudio de 2.300 fotografías y 308 minutos de video, realizados bajo el consentimiento informado de la institución y los participantes del estudio.

Para la aplicación de la metodología, se comprendió el cuerpo como un espacio social del individuo con el cual experimenta el mundo; por esto, el cuerpo debe ser entendido en la lectura de esta investigación como un lugar (Mcdowell, 1999) donde se inscriben significados, esto reconociendo que el cuerpo de la investigadora fue el instrumento de investigación, filtrando los resultados y análisis que ahora son presentados. Este reconocimiento hace parte de una exigencia metodológica propia de la etnografía, que busca procesos de reflexividad en los que quien observa, también se observe a sí mismo.

\section{Resultados}

\section{La institución y su cuerpo}

El Ejército es una de las fuerzas armadas del país. Podría decirse que es la que cuenta con mayor protagonismo no solo por el conflicto armado nacional, sino por las experiencias cercanas que tiene la población civil con este, como la prestación del servicio militar obligatorio y la militarización urbana.

Esta organización, como cualquier otra fuerza pública, tiene la facultad de ejercer el monopolio de la fuerza de manera legal y, a causa de esta 
facultad, estas instituciones estatales tienen básicamente dos funciones: guardar el orden interno y preparar al país en caso de guerra (Rueda, 1968).

Los y las militares, como funcionarios del Ejército, deben aprender las formas de ejercer la fuerza y el orden dentro del país; es por esto que el Ejército cuenta con instituciones educativas que permiten la adquisición de estos conocimientos. Entre las instituciones más importantes están la Escuela Militar de Cadetes José María Córdova (ESMIC) y la Escuela Militar de Suboficiales "Sargento Inocencio Chincá", estas instituciones tienen sus extensiones en fuertes militares, campos de entrenamiento y batallones.

La presente investigación tuvo como lugar la Escuela Militar de Cadetes José María Córdova (ESMIC) en Bogotá. Esta institución tiene como función principal la formación de los y las oficiales que trabajarán para el Ejército. Los y las oficiales son comandantes de pelotón ${ }^{2}$, profesionales en ciencias militares y disciplinas complementarias ${ }^{3}$; los cadetes que se convertirán en oficiales son jóvenes entre los 16 y 21 años, quienes voluntariamente deciden aprender la profesión militar que podría compararse, en sus justas proporciones, a una carrera universitaria civil. El título de oficial es producto de la formación adquirida desde un proceso educativo intenso, que es recibido durante cuatro años dentro de la ESMIC.

$\mathrm{Al}$ ingresar a la ESMIC, los y las jóvenes adquieren el título de cadetes; esto es, el primer grado dentro de la línea de mando militar ${ }^{4}$, es decir que son los subordinados de los otros grados de oficiales militares. La línea de mando está encabezada por el presidente del país, el ministro de Defensa, el comandante de las Fuerzas Militares y el comandante del Ejército Nacional; seguido de estos están los militares pertenecientes a los diferentes grados de oficiales del Ejército.

Desde los resultados de este trabajo es posible entender la construcción del cuerpo en una institución con unas condiciones fijadas desde sus inicios para el adiestramiento, el disciplinamiento y la corrección de los cuerpos de

2 Organización militar en la que está al mando un oficial, cuatro suboficiales y 36 soldados.

3 La Escuela Militar ofrece carreras complementarias en: Administración Logística, Derecho, Relaciones Internacionales y Educación Física.

4 Los cadetes pertenecen al primer grado de la línea de mando militar sin contar con otros subordinados que tienen la misma condición de subordinación como los soldados bachilleres y soldados profesionales. 
los futuros oficiales del Ejército; estos procesos vividos por cada sujeto dentro de la institución se inscriben en lo que Mauss (1971) llama la primera herramienta del ser humano: el cuerpo.

Desde este primer momento en el proceso de incorporación, los futuros cadetes y cadetas comprenden desde su experiencia corporal el lugar al que están llegando y las exigencias que este les va a demandar. El cuerpo del cadete $^{5}$ se entiende como el lugar que le permite comprender el mundo y habitarlo (Cabra, 2006). En el caso de los y las cadetes, el cuerpo se convierte en un receptor activo de prácticas y símbolos (Cruz, 2006) que durante los siguientes cuatro años de su formación se incorporarán casi que de manera natural.

El cuerpo, como una herramienta institucional y socialmente productiva (Foucault, 1975), es administrado por la institución en busca de la disposición del cadete para el aprendizaje de nuevas prácticas necesarias para la vida militar; de esta manera, la institución piensa en el cultivo de cierto tipo de cualidades que el cadete debe apropiar para que, al terminar su proceso de formación en la ESMIC, pueda reproducir lo aprendido en el manejo de sus tropas y en el adiestramiento de futuros oficiales.

Las instituciones modelan los cuerpos que tienen a su cargo según las necesidades propias de cada escenario; esto es posible si se entiende que el cuerpo puede ser un objeto que se manipula, se educa, se le da forma, que obedece y que responde como si fuera una máquina (Foucault, 1975); de esta manera, el cuerpo se produce en consecuencia de los espacios y los objetos a los que es expuesto. Es posible pensar entonces que la institución cuenta con un cuerpo propio conformado de espacios, olores, formas y estímulos que contienen los cuerpos de los sujetos que con el tiempo se convertirán en extensiones del cuerpo de la institución, incluso al estar fuera de ella.

\section{Intervención física y social del cuerpo del cadete}

El cuerpo de los sujetos que llega a la formación militar no es expuesto por primera vez al disciplinamiento propio de la institución, sino que muchos de estos cuerpos han conocido experiencias similares en otros lugares que

5 Utilizo la noción de "el cadete" para referirme a los hombres y mujeres que son distinguidos dentro de la institución bajo este rango, la mayoría de población que pude identificar son personas blanco mestizas de clase media de diferentes regiones del país. 
conservan muchas de las lógicas militares, como los colegios, los hospitales, los conventos, las prisiones y las fábricas, además de conocer innumerables representaciones de la vida y de los cuerpos militares en las películas, los videojuegos, las telenovelas, la moda, los cómics, la música, la literatura, entre muchas otras que funcionan como tecnologías de construcción del mundo militar y contribuyen a la seducción temprana por este tipo de oficio.

La intervención del cuerpo militar se hace por medio de la tecnología, entendida como un conjunto de conocimientos en favor de suplir las necesidades humanas. Las tecnologías se pueden ver traducidas en ciencias, técnicas y artefactos materiales, por tanto, estas son productos históricos y culturales.

La formación del cuerpo de los cadetes está intervenida por medio de diferentes tipos de tecnologías, entre ellas las que se encuentran en el aprendizaje de las ciencias militares, donde se incluye el aprender a mimetizarse ${ }^{6}$; a utilizar diferentes tipos de armas, elementos de comunicación y de ubicación; estrategias de combate y de inteligencia militar, entre otros conocimientos.

El desarrollo y acceso a la tecnología hacen la diferencia entre los ejércitos; aunque es importante destacar que existen otras tecnologías menos evidentes en la intervención del cuerpo del cadete que hacen parte activa de su construcción, por ejemplo las tecnologías como el cine o los videojuegos, que se encargan de reproducir discursos institucionalizados de cómo deben ser representados los cuerpos en relación al sexo, la edad y la clase social.

Foucault (1990) expone la existencia de cuatro tipos principales de tecnologías que influyen en la construcción de los sujetos sociales: las tecnologías de producción, las tecnologías de sistemas de signos, las tecnologías del poder y las tecnologías del yo; para analizar la situación de los cadetes, me interesa esta última, aunque todas trabajan en conjunto.

Las tecnologías del yo son las que "permiten a los individuos efectuar, por cuenta propia o con ayuda de otros, cierto número de operaciones sobre su cuerpo y su alma, pensamientos, conductas, o cualquier forma de ser" (Foucault, 1990, p. 48). Todas las tecnologías mencionadas por Foucault

6 Los cadetes aprenden durante sus instrucciones militares a maquillarse la cabeza y las manos con pinturas de mimetismo, además de construir prendas con ramas, hojas y retazos de tela que les permiten camuflarse en los escenarios donde tendrán que patrullar en un futuro. 
están relacionadas con la modificación y el aprendizaje de cada individuo por medio de la adquisición de habilidades y actitudes.

Estos procesos, en los cuales actúan las tecnologías sobre los sujetos, se pueden ver dentro de la institución en las formas en que cambian las maneras utilizadas por los antiguos civiles para convertirse en militares; estas maneras, estas formas y estas disposiciones se pueden observar en lo que Mauss (2006) denomina "técnicas corporales", y en un concepto más amplio en lo que Bourdieu (2007) reconoce como habitus. Estas formas de ser en el mundo militar forman al sujeto de manera integral, puesto que los procesos que tienen como objeto el cuerpo material también influencia la percepción simbólica del cuerpo en diferentes dimensiones (mental, espiritual, sexual, entre otras) que tienen efectos reales en la vida de los sujetos.

\section{Aplicación de técnicas para la modificación corporal del cadete}

En la madrugada, los cadetes inician su día, que previamente ha sido planeado por la institución, con una actividad para cada hora del día — que básicamente se divide en comer, asistir a clases en aulas, asistir a instrucción militar y trotar-. Todos los momentos y acciones que deben ejecutar los cadetes son enseñados a partir de estímulos visuales como el reconocimiento de la autoridad por medio de la lectura del uniforme a la hora de saludar, y estímulos sonoros como el reconocimiento de las voces de mando, los toques de trompeta y los himnos.

Por ejemplo, el reconocido toque de diana, que se utiliza en las unidades militares para indicar el momento en que se inician las actividades, tiene una duración de quince minutos y es precedido por la voz de mando: "Buenos días compañía de pie, desde este momento tienen quince minutos de diana", el tiempo que deben durar los cadetes realizando sus primeras actividades del día, entre las que se encuentran bañarse, vestirse, ordenar el alojamiento y presentarse a la formación. Los sonidos militares son los mayores estímulos, según la observación, para reaccionar frente a la autoridad; los cuerpos de los cadetes entienden estos sonidos como estímulos que

7 Unidad militar compuesta, en promedio, por cuatro pelotones de treinta y seis personas cada uno. 
les indican cómo y cuándo moverse, produciendo en los cuerpos movimientos automáticos.

Dentro de estas tecnologías también se encuentran la disposición de los espacios de la arquitectura castrense, las formas de desplazamiento militar como la marcha y el trote, la construcción de la sexualidad de los cadetes por medio de la regulación, prohibición y vigilancia de las relaciones sexuales, el ejercicio físico, el buen porte del uniforme, los castigos, entre otras.

\section{Gráfico 1. Cadetes en formación}

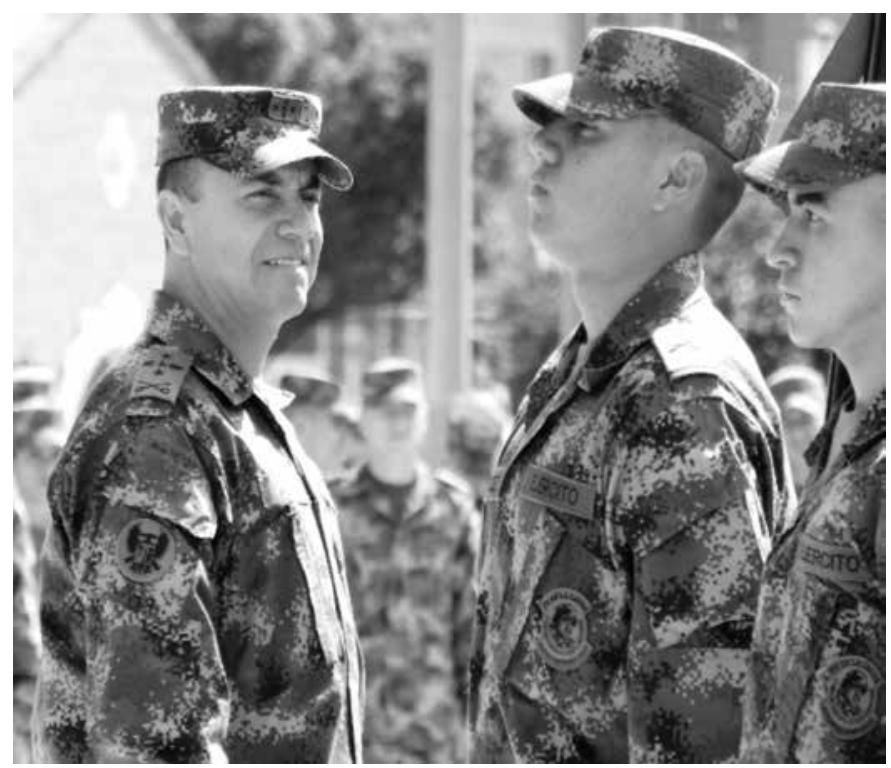

Fuente: fotografía tomada por la autora (22/07/14).

En el Gráfico 1 se pueden observar a los cadetes en formación con la postura de su cuerpo totalmente ordenada según los estándares institucionales: estar erguido, tener la vista al frente y los brazos a los lados. Se puede observar cómo reaccionan los cuerpos en el momento de la supervisión de un coronel, que es reconocido automáticamente en la institución por sus distintivos y por otros símbolos propios del don de mando ${ }^{8}$ adquirido a lo

8 Capacidad militar adquirida a través de la formación y ascenso en la institución que 
largo de la carrera militar, entre los cuales se incluye su manera de caminar, de hablar, de mirar y en general de mandar; todos estos relacionados directamente con las disposiciones propias del cuerpo, que se continúan adquiriendo con el paso del tiempo y el ascenso social dentro de la institución. En el caso de los oficiales de alto rango, como el que se observa en el Gráfico 1, es aún más visible la intervención de la institución en el cuerpo.

La ESMIC, como escuela, es un lugar diseñado en sus espacios y técnicas disciplinarias para el aprendizaje de reglas que enseñan a los sujetos a respetar el puesto que están destinados a ocupar: siempre como subordinados de la línea de mando military respetando una división social del trabajo, que en la ESMIC se hace por medio de la división en las armas, las profesiones y los grados militares.

Las jerarquías propias del Ejército también hacen parte de una dominación de clase, ya que el ascenso en las líneas de mando también implica un ascenso social dentro de la comunidad militar, que se acompaña con un mejor salario, el aumento del don de mando — teniendo más subordinados a cargo - y ocupando empleos socialmente más reconocidos dentro de las diferentes unidades militares.

La ESMIC, como constructora del cuerpo de los cadetes, es un lugar que permite entender cómo la ideología estatal se puede reproducir en la educación para el predominio de la clase dominante. El sistema militar funciona casi de manera perfecta para la reproducción de la fuerza de trabajo, que básicamente exige la posibilidad de tener vivienda, vestido, alimentación y todas las condiciones que le permiten a un sujeto continuar trabajando (Althusser, 1969).

En la formación militar, los cadetes aprenden a necesitar cada vez menos de lo que cree necesitar una persona civil, pues se acostumbran a compartir baños y alojamientos, y a no salir de la institución, lo que permite que su horario laboral se extienda y que ahorren tiempo en desplazamientos; aprenden a comer, según las horas y momentos establecidos por la institución, la comida ofrecida por esta; el vestido se reduce a la dotación anual de uniformes; aprenden a hacer todo a gran velocidad; se inculcan los valores

se caracteriza por la eficacia al momento de dar órdenes y liderar al personal militar, esta capacidad es propia en la ESMIC de los oficiales militares y no del personal administrativo, aunque este porte el uniforme y los rangos militares. 
del ejercicio, que mantienen los cuerpos saludables, junto con la revisión constante del cuerpo en pruebas físicas y médicas; se enseña a los cadetes a ser resistentes al hambre, al frío, al cansancio, incluso a sus sentimientos, por lo cual los cadetes como oficiales terminan siendo unos de los mejores trabajadores del país, quizá unos de los que más le ahorran recursos a la institución empleadora.

En términos de Althusser (1969), la escuela, junto con otras instituciones del Estado - como el Ejército, la iglesia y la familia-, enseñan habilidades que aseguran el sometimiento a la ideología dominante y validan el dominio de sus prácticas. Las disposiciones a la obediencia y el sometimiento, en el caso de los militares, viene reforzada por la naturalización que existe de la dominación dentro de la milicia, que se puede observar en los falsos estereotipos que se tienen de los militares del Ejército, como hombres rudos, grandes, fuertes, groseros, hipermasculinizados, blancos, musculosos, sucios, brutos, entre muchos otros que el lector ya conocerá.

\section{Consideraciones finales}

En síntesis, a la pregunta: ¿cómo prepara un cadete su cuerpo para el oficio militar?, se podría responder que en su experiencia hay tres momentos importantes que influencian la construcción de su cuerpo:

- Un primer momento donde predispone su cuerpo al considerar al Ejército como opción de vida: el cadete acepta desde lo que conoce como las representaciones de la vida militar o las experiencias cercanas a la milicia, las condiciones que comúnmente se reconocen como propias de la vida castrense, como la subordinación, la obediencia y la exigencia física. Esta predisposición está construida desde la formación por parte de otras instituciones que han intervenido el cuerpo de los jóvenes anteriormente, dotándolos de técnicas y formas de ser en el mundo que coinciden con las exigencias sociales propias de la ESMIC y que influencian el reconocimiento de las aptitudes de un sujeto.

- Un segundo momento, donde al incorporarse a la institución, el cadete pone a prueba sus capacidades de adaptación a la vida militar, reconociendo nuevos espacios, nuevas normas de comportamiento y, en general, nuevas exigencias sociales propias del oficio que está aprendiendo 
y que le obliga a cambiar muchas de sus prácticas anteriores como civil. En este segundo momento, los cadetes son permeados por los espacios de la institución, las distinciones sociales, el adiestramiento de sus cuerpos y los símbolos propios de las competencias léxicas del Ejército.

- Finalmente, un tercer momento, donde el cadete logra adaptarse a la vida militar, adoptando las formas de vida castrense y, lo más importante: reproduciéndolas con otros cadetes. Este tercer momento se exhibe ceremonialmente cuando la institución reconoce al sujeto como subteniente, lo que significa ser un profesional de las armas, tener el don de mando y estar listo para comandar a otros militares que deben adoptar sus mismas formas.

Las condiciones que propone la ESMIC en la formación de los cuerpos de los cadetes no solo hacen parte del interés de producir buenos oficiales: como otras instituciones, el Ejército tiene unos vínculos sociales más grandes que permiten también la creación de trabajadores con la cualidad de reproducir su fuerza de trabajo y, además, de obedecer y reproducir el proceso por medio del arte de mandar (Gavet, 1944).

El Ejército interviene directamente como fuerza represiva de apoyo al Estado y quizá sea por esto que ostente uno de los mejores grupos de obreros que pueda tener una empresa, ya que el Ejército casi logra ser autosuficiente. Los mismos miembros de la ESMIC limpian las instalaciones; los soldados prestan servicios de cafetería; los cadetes manejan los turnos de vigilancia de la ESMIC; los alféreces supervisan a los cadetes; los militares hacen parte del profesorado, entre muchas otras condiciones que hacen que el Ejército en general sea una institución muy cerrada y autónoma.

Las Fuerzas Armadas cuentan con lugares exclusivos para ellas, donde se pueden observar a las personas actuando en una sociedad paralela y muy distinta a la sociedad civil. Estos lugares son interesantes por sus maneras propias de funcionar como una subcultura nacional que, por las condiciones actuales del país, se enfrenta a procesos de modernización dignos de ser estudiados.

El cuerpo, como instrumento de trabajo y de socialización permanente con el mundo, permite encontrar desde el estudio de su transformación relaciones macrosociales de las diferentes comunidades a las que este ha sido expuesto, una reflexión continua sobre el cuerpo puede pensarse en equivalencia a una reflexión sobre la sociedad y sus cambios estructurales. 
Se puede reconocer, producto de la socialización de este trabajo, que el tema militar produce escozor en muchas personas, incluyendo a investigadores sociales que tienen una aversión a la disciplina, la violencia, el Estado y los demás aspectos que implican a las Fuerzas Armadas; sin embargo, este trabajo pretende hacer una invitación para que se conozca más de estos grupos y se abarquen temas socialmente interesantes más allá de los estereotipos militares y los temas relacionados a la violencia y el conflicto, además de que permiten pensar también la formación del cuerpo en otro tipo de instituciones donde conviven los civiles y donde difícilmente se reconocen las formas de intervención corporal.

\section{Referencias}

Althusser, L. (1969). Ideología y aparatos ideológicos de estado. Bogotá: Tupac - Amaru.

Ayús, R., y Eroza, E. (2008). El cuerpo y las ciencias sociales. Revista Pueblos y Fronteras (4), pp. 1 - 56.

Bourdieu, P. (2007). El sentido práctico. Buenos Aires: Siglo Veintiuno Editores.

Cabra, N. (2006). Comunicación o las resonancias del cuerpo. XII Encuentro

Latinoaméricano de Facultades de Comunicación Social FELAFACS. Bogotá,

Pontificia Universidad Javeriana.

Castañeda, A. (2006). El Ejercito: ¿El reflejo más bello del modelo Patriarcal? Bogota: Pontificia Universidad Javeriana.

Cruz, S. (2006). Cuerpo, masculinidad y jóvenes. Iberoforum. Revista de Ciencias

Sociales de la Universidad Iberoamericana, I(I), 1-9.

De Lauretis, T. (1989). Technologies of gender. Essays on Theory, Film and Fiction. Londres: Macmillan Press.

Foucault, M. (1975). Vigilar y Castigar. Madrid: España Editores.

Foucault, M. (1990). Tecnologías del yo y otros textos afines. Barcelona: Paidos.

Gavet, A. (1944). El arte de mandar principios del mando para uso de los oficiales de todo grado. Santiago: Instituto Geográfico Militar.

López, A. (1996). Cuerpo Humano e Ideología. México: Universidad Nacional Autónoma de México.

Mauss, M. (1971). Sociología y antropología. Madrid: Tecnos. 
Mauss, M. (2006). Manual de Etnografía. Buenos Aires: Fondo De Cultura Económica de Argentina.

McDowell, L. (1999). Gender, identity \& place understanding feminist geographies. Minnesota: University of Minnesota Press.

Montgomery, B. (1969). Historia del Arte de la Guerra. Madrid: Aguilar Ediciones S. A.

Pedraza, Z. (2007). Políticas y Estéticas del Cuerpo en América Latina. Bogotá: Universidad de los Andes, Facultad de Ciencias Sociales, Departamento de Antropología, Centro de Estudios Socioculturales e Internacionales - CESO.

Rueda, T. (1968). El Ejército Nacional. Bogotá: Escuela Superior de Guerra de Colombia.

Torres Del Río, C. (2000). Fuerzas Armadas Y Seguridad Nacional. Bogotá: Planeta. 